\title{
The lack of impact of the 'impact factor' in clinical medicine
}

\author{
Peter E Lipsky
}

Nature Clinical Practice Rheumatology (NCPR) will not have an impact factor (IF) for over 2 years. But does NCPR actually want an IF (we have no choice), and what will it signify? The IF is widely used to assess the supposed influence of research articles on the course of scientific investigation. Our 2008 IF will be calculated by counting all the citations in 2008 of NCPR articles published in 2006 and 2007, and dividing this value by the number of NCPR articles published in 2006 and 2007. The IF was envisioned over 50 years ago with the purpose of eliminating "the uncritical citation of fraudulent, incomplete or obsolete data by making it possible for the conscientious scholar to be aware of criticisms of earlier work" (Garfield E [1955] Science 122: 108-111). The IF has subsequently morphed into an institutionalized means of ranking the quality of scientific journals and, by implication, the individual articles published within them; for researchers, the IF influences employability, promotion, grant acceptability and bonus payments, and has been likened to a popularity contest.

A major concern with the IF in its current iteration is that it is administered by a 'for-profit' company (Thomson Scientific), which selects the articles to be included in the denominator of the equation according to a secret formula that apparently can be influenced by negotiation (PLosMed [2006] 3: e291). Additionally, journals can manipulate their content to improve their IF, and there is no monitoring of the appropriateness of a citation, or the reason for a citation: an erroneous paper could be frequently cited and thereby bolster the IF. Finally, IFs apply to journals and not to individual articles-since citation rates vary greatly in different scientific fields and review articles

\section{The IF is \\ neither a \\ surrogate for \\ quality, nor an \\ estimate of \\ the influence \\ of an article \\ on clinical \\ practice}

\section{PE Lipsky is \\ the Editor-in- \\ Chief of Nature \\ Clinical Practice \\ Rheumatology.}

\section{Competing interests}

The author declared he has no competing interests.

www.nature.com/clinicalpractice doi:10.1038/ncprheum0462 contribute disproportionately, the number of citations of an individual article might not reflect the IF of an entire journal. Another of Garfield's goals, "to evaluate the significance of a particular work and its impact on the literature and thinking of the period" is clearly not being met by the current system.

If the IF is problematic in basic science, it is even more so in clinical medicine: calculation of the IF necessitates that the target audience actually contributes routinely to the literature. Since busy clinicians rarely have time to write articles, it is obvious that the IF cannot be used to gauge the importance of an article. The IF is neither a surrogate for quality, nor an estimate of the influence of an article on clinical practice. The clinical community already knows this, since IFs demonstrably do not correlate with the subjective views of individuals in numerous clinical disciplines (Lewison G [2002] Scientometrics 53: 229-240).

NCPR is committed to communicating the latest research developments to rheumatologists in an informative and useful way. Our content is organized to accomplish that goal, and our choice of subjects is based on the perceived needs and interests of our readers. Since our mission is clear, and we believe the IF is irrelevant to our success in this regard, we have elected to do little to bolster our IF. True, we publish reviews, but this activity is done to meet our aims, not to enhance our IF. We do not feel that the current IF will reflect either our quality or our potential influence on clinical practice. Only if a new validated metric is developed that can evaluate the true quality and value of journal articles can we make real progress in improving the communication of new information in clinical medicine. 